\title{
Generalized Quasi Variational-Type Inequalities
}

\author{
Mohammad Kalimuddin Ahmad, Salahuddin \\ Department of Mathematics, Aligarh Muslim University, Aligarh, India \\ Email: ahmad_kalimuddin@yahoo.co.in, salahuddin12@mailcity.com
}

Received September 9, 2011; revised November 4, 2011; accepted November 12, 2011

\begin{abstract}
In this paper, we define the concepts of $(\eta, h)$-quasi pseudo-monotone operators on compact set in locally convex Hausdorff topological vector spaces and prove the existence results of solutions for a class of generalized quasi variational type inequalities in locally convex Hausdorff topological vector spaces.
\end{abstract}

Keywords: Generalized Quasi Variational Type Inequalities (GQVTI); $(\eta, h)$-Quasi Pseudo-Monotone Operator; Locally Convex Hausdorff Topological Vector Spaces; Compact Sets; Bilinear Functional; Lower Semicontinuous; Upper Semicontinuous

\section{Introduction}

Variational inequality theory has appeared as an effective and powerful tool to study and investigate a wide class of problems arising in pure and applied sciences including elasticity, optimization, economics, transportation, and structural analysis, see for instance [1,2]. In 1966, Browdev [3] first formulated and proved the basic existence theorems of solutions to a class of nonlinear variational inequalities. In 1980, Giannessi [1] introduced the vector variational inequality in a finite dimensional Euclidean space. Since then Chen et al. [4] have intensively studied vector variational inequalities in abstract spaces and have obtained existence theorems for their inequalities.

The pseudo-monotone type operators was first introduced in [5] with a slight variation in the name of this operator. Later these operators were renamed as pseudomonotone operators in [6]. The pseudomonotone operators are set-valued generalization of the classical pseudomonotone operator with slight variations. The classical definition of a single-valued pseudo-monotone operator was introduced by Brezis, Nirenberg and Stampacchia [7].

In this paper we obtained some general theorems on solutions for a new class of generalized quasi variational type inequalities for $(\eta, h)$-quasi-pseudo-monotone operators defined as compact sets in topological vector spaces. We have used the generalized version of Ky Fan's minimax inequality [8] due to Chowdhury and Tan [9].

Let $X$ and $Y$ be the topological spaces,

$T: X \rightarrow 2^{Y}$ be the mapping and the graph of $T$ is the set $G(T)=\{(x, y) \in X \times Y: y \in T(x)\}$. In this paper, $\Phi$ denotes either the real field $\mathbb{R}$ or the complex field $\mathbb{C}$. Let $E$ be a topological vector space over $\Phi, F$ be a vector space over $\Phi$ and $\langle\cdot, \cdot\rangle: F \times E \rightarrow \Phi$ be a bilinear functional.

For each nonempty subset $A$ of $E$ and $\varepsilon>0$, let $W\left(x_{0} ; \varepsilon\right)=\left\{y \in F:\left|\left\langle y, x_{0}\right\rangle\right|<\varepsilon\right\}$ and $U(A ; \varepsilon)=\left\{y \in F: \sup _{x \in A}|\langle y, x\rangle|<\varepsilon\right\}$ for $x_{0} \in E$. Let $\sigma(F, E)$ be the (weak) topology on $F$ generated by the family $\{W(x ; \varepsilon): x \in E$ and $\varepsilon>0\}$ as a subbase for the neighbourhood system at 0 and $\delta\langle F, E\rangle$ be the (strong) topology on $F$ generated by the family $\{U(A ; \varepsilon): A$ is a nonempty bounded subset of $E$ and $\varepsilon>0\}$ as a base for the neighbourhood system at 0 . The bilinear functional $\langle\cdot, \cdot\rangle: F \times E \rightarrow \Phi$ separates points in $F$, i.e., for each $0 \neq y \in F$, there exists $x \in E$ such that $\langle y, x\rangle \neq 0$, then $F$ also becomes Hausdorff. Furthermore, for a net $\left\{y_{\alpha}\right\}_{\alpha \in \Gamma}$ in $F$ and for $y \in F$,

1) $y_{\alpha} \rightarrow y$ in $\sigma\langle F, E\rangle$ if and only if $\left\langle y_{\alpha}, x\right\rangle \rightarrow\langle y, x\rangle$ for each $x \in E$ and

2) $y_{\alpha} \rightarrow y$ in $\sigma\langle F, E\rangle$ if and only if $\left\langle y_{\alpha}, x\right\rangle \rightarrow\langle y, x\rangle$ uniformaly for $x \in A$ for each nonempty bounded subset $A$ of $E$.

Given a set-valued map $S: X \rightarrow 2^{X}$ and two set valued maps $M, T: X \rightarrow 2^{F}$, the generalized quasi variational type inequality (GQVTI) problem is to find $\hat{y} \in X$ and $\hat{w} \in T(\hat{y})$ such that $\hat{y} \in S(\hat{y})$ and

$$
\begin{aligned}
& \operatorname{Re}\langle f-\hat{w}, \eta(\hat{y}, x)\rangle \leq 0, \\
& \text { for all } x \in S(\hat{y}) \text { and } f \in M(\hat{y}),
\end{aligned}
$$

where $\eta: X \times X \rightarrow E$.

If $\eta(\hat{y}, x)=\hat{y}-x$, then generalized quasi variational type inequality (GQVTI) is equivalent to generalized quasi variational inequality (GQVI). 
Find $\hat{y} \in X$ and $\hat{w} \in T(\hat{y})$ such that $\hat{y} \in S(\hat{y})$ and

$$
\operatorname{Re}\langle f-\hat{w}, \hat{y}-x\rangle \leq 0 \text { for all } x \in S(y)
$$

and $f \in M(\hat{y})$ was introduced by Shih and Tan [10] in 1989 and later was stated by Chowdhury and Tan in [11].

Definition 1. Let $X$ be a nonempty subset of a topological vector space $E$ over $\Phi$ and $F$ be a topological vector space over $\Phi$, which is equipped with the $\sigma(F, E)$-topology. Let $\langle\cdot, \cdot\rangle: F \times E \rightarrow \Phi$ be a bilinear functional. Suppose we have the following four maps.

1) $h: X \times X \rightarrow \mathbb{R}$

2) $\eta: X \times X \rightarrow E$

3) $M: X \rightarrow 2^{F}$

4) $T: X \rightarrow 2^{F}$.

1) Then $T$ is said to be an ( $\eta, h)$-quasi pseudo-monotone type operator if for each $y \in X$ and every net $\left\{y_{\alpha}\right\}_{\alpha \in \Gamma}$ in $X$ converging to $y$ (or weakly to $y$ ) with

$\limsup \left[\inf _{f \in M(y)} \inf _{u \in T\left(y_{\alpha}\right)} \operatorname{Re}\left\langle f-u, \eta\left(y_{\alpha}, y\right)\right\rangle+h\left(y_{\alpha}, y\right)\right] \leq 0$.

We have

$$
\begin{aligned}
& \limsup \left[\inf _{f \in M(x)} \inf _{u \in T\left(y_{\alpha}\right)} \operatorname{Re}\left\langle f-u, \eta\left(y_{\alpha}, x\right)\right\rangle+h\left(y_{\alpha}, x\right)\right] \\
& \geq \inf _{f \in M(x)} \inf _{w \in T(y)} \operatorname{Re}\langle f-w, \eta(y, x)\rangle+h(y, x),
\end{aligned}
$$

for all $x \in X$;

2) $T$ is said to be $h$-quasi-pseudomonotone operator if $T$ is $(\eta, h)$-quasi-pseudomonotone operator with $\eta(x, y)=x-y$ and for some $h^{\prime}: X \rightarrow \mathbb{R}$,

$$
h(x, y)=h^{\prime}(x)-h^{\prime}(y) \text { for all } x, y \in X .
$$

3) a quasi-pseudo monotone operator if $T$ is an $h$-quasi pseudo-monotone operator with $h \equiv 0$.

Remark 1. If $M \equiv 0$ and $T$ is replaced by $-T$, then $h$-quasi-pseudo monotone operator reduces to the $h$-pseudo monotone operator, see for example [5]. The $h$-pseudo monotone operator defined in [5] is slightly more general than the definition of $h$-pseudo monotone operator given in [12]. Also we can find the generalization of quasi-pseudo monotone operator in [11] and for more detail see [13].

Theorem 1. [8] Let $E$ be a topological vector space, $X$ be a nonempty convex subset of $E$ and $f: X \times X \rightarrow \mathbb{R} \cup\{-\infty,+\infty\}$ be such that

1) For each $A \in F(X)$ and each fixed $x \in c o(A)$, $y \rightarrow f(x, y)$ is lower semicontinuous on $\operatorname{co}(A)$;

2) For each $A \in F(X)$ and each $y \in c o(A)$, $\min f(x, y) \leq 0$;

${ }^{x} \in \mathcal{A}_{\text {) For each }} A \in F(X)$ and each $x, y \in c o(A)$, every net $\left\{y_{\alpha}\right\}_{\alpha \in \Gamma}$ in $X$ converging to $y$ with $f\left(t x+(1-t) y, y_{\alpha}\right) \leq 0$ for all $\alpha \in \Gamma$ and all $t \in[0,1]$ we have $f(x, y) \leq 0$;

4) There exist a nonempty closed compact subset $K$ of $X$ and $x_{0} \in K$ such that

$$
f\left(x_{0}, y\right)>0 \text { for all } y \in X \backslash K .
$$

Then there exists $\hat{y} \in K$ such that

$$
f(x, \hat{y}) \leq 0 \text { for all } x \in X .
$$

\section{Preliminaries}

In this section, we shall mainly state some earlier work which will be needed in proving our main results.

Lemma 1. [14] Let $X$ be a nonempty subset of a Hausdorff topological vector space $E$ and $S: X \rightarrow 2^{E}$ be an upper semicontinuous map such that $S(x)$ is a bounded subset of $E$ for each $x \in X$. Then for each continuous linear functional $p$ on $E$, the map $f_{p}: X \rightarrow \mathbb{R}$ defined by

$$
f_{p}(y)=\sup _{x \in S(y)} \operatorname{Re}\langle p, x\rangle \text { is upper semicontinuous i.e., }
$$

for each $\lambda \in R$,

the set $\left\{y \in X: f_{p}(y)=\sup _{x \in S(y)} \operatorname{Re}\langle p, x\rangle<\lambda\right\}$ is open in $X$.

Lemma 2. [15] Let $X, Y$ be topological spaces, $f: X \rightarrow \mathbb{R}$ be non-negative and continuous and $g: Y \rightarrow \mathbb{R}$ be lower semicontinuous. Then the map $F: X \times Y \rightarrow \mathbb{R}$, defined by $F(x, y)=f(x) g(y)$ for all $(x, y) \in X \times Y$, is lower semicontinuous.

Lemma 3. [11] Let $E$ be a topological vector space over $\Phi, X$ be a nonempty compact subset of $E$ and $F$ be a Hausdorff topological vector space over $\Phi$. Let $\langle\cdot, \cdot\rangle: F \times E \rightarrow \Phi$ be a bilinear functional and

$T: X \rightarrow 2^{F}$ be an upper semicontinuous map such that each $T(x)$ is compact. Let $M$ be a nonempty compact subset of $F, x_{0} \in X$ and $h: X \rightarrow \mathbb{R}$ be continuous. Define $g: X \rightarrow \mathbb{R}$ by

$$
g(y)=\left[\inf _{f \in M(x)} \inf _{w \in T(y)} \operatorname{Re}\left\langle f-w, y-x_{0}\right\rangle\right]+h(y)
$$

for each $y \in X$.

Suppose that $\langle\cdot, \cdot\rangle$ is continuous on the (compact) subset $\left[M-\bigcup_{y \in X} T(y)\right] \times X$ of $F \times E$. Then $g$ is lower semicontinuous on $X$.

Lemma 4. [11] Let $E$ be a topological vector space over $\Phi, F$ be a vector space over $\Phi$ and $X$ be a nonempty convex subset of $E$. Let $\langle\cdot, \cdot\rangle: F \times E \rightarrow \Phi$ be a bilinear functional, equip $F$ with the $\sigma\langle F, E\rangle$ - 
topology. Let $h: X \times X \rightarrow \mathbb{R}$ be convex with second argument and $h(x, x)=0$ for all $x \in X$. Let $M: X \rightarrow F$ be lower semicontinuous along line segments in $X$ to the $\sigma\langle F, E\rangle$-topology on $F$. Let $S: X \rightarrow 2^{X}$ and $T: X \rightarrow 2^{F}$ be two maps. Let the continuous map $\eta: X \times X \rightarrow E$ be convex with second argument, $\eta(x, x)=0$ for every $x \in X$. Suppose that there exists $\hat{y} \in X$ such that $\hat{y} \in S(\hat{y}), S(\hat{y})$ is convex and

$$
\begin{aligned}
& \inf _{f \in M(x)} \inf _{w \in T(y)} \operatorname{Re}\langle f-w, \eta(\hat{y}, x)\rangle+h(x, \hat{y}) \leq 0 \\
& \text { for all } x \in S(\hat{y}) .
\end{aligned}
$$

Then

$$
\begin{aligned}
& \inf _{f \in M(\hat{y})} \inf _{w \in T(\hat{y})} \operatorname{Re}\langle f-w, \eta(\hat{y}, x)\rangle+h(x, \hat{y}) \leq 0 \\
& \text { for all } x \in S(\hat{y}) .
\end{aligned}
$$

Theorem 2. [16] Let $X$ be a nonempty convex subset of a vector space and $Y$ be a nonempty compact convex subset of a Hausdorff topological vector space. Suppose that $f$ is a real-valued function on $X \times Y$ such that for each fixed $x \in X$, the map $y \rightarrow f(x, y)$, i.e., $f(x, \cdot)$ is lower semicontinuous and convex on $Y$ and for each fixed $y \in Y$, the map $x \rightarrow f(x, y)$, i.e., $f(\cdot, y)$ is concave on $X$. Then

$$
\min _{y \in Y} \sup _{x \in X} f(x, y)=\sup _{x \in X} \min _{y \in Y} f(x, y) \text {. }
$$

\section{Existence Result}

In this section, we prove the existence theorem for the solutions to the generalized quasi variational type inequalities for $(\eta, h)$-quasi-pseudo monotone operator with compact domain in locally convex Hausdorff topological vector spaces.

Theorem 3. Let $E$ be a locally convex Hausdorff topological vector space over $\Phi, X$ be a nonempty compact convex subset of $E$ and $F$ a Hausdorff topological vector space over $\Phi$. Let $\langle\cdot, \cdot\rangle: F \times E \rightarrow \Phi$ be a bilinear continuous functional on compact subset of $F \times X$. Suppose that

1) $S: X \rightarrow 2^{X}$ is upper semicontinuous such that each $S(x)$ is closed and convex;

2) $h: X \times X \rightarrow \mathbb{R}$ is convex with second argument, $h(\cdot, x)$ is lower semicontinuous and $h(x, x)=0$ for $x \in X$;

3) $\eta: X \times X \rightarrow E$ is convex with second argument, $\eta(\cdot, y)$ is continuous and $\eta(x, x)=0$ for all $x \in X$;

4) $T: X \rightarrow 2^{F}$ is an $(\eta, h)$-quasi-pseudo-monotone operator and is upper semicontinuous such that each $T(x)$ is compact, convex and $T(X)$ is strongly bounded;

5) $M: X \rightarrow F$ is a linear and upper semicontinuous map in $X$ such that each $M(x)$ is (weakly) compact convex;

6) the set

$$
\begin{aligned}
\Sigma=\{y & \in X: \sup _{x \in S(y)} \inf _{w \in T(y)} \operatorname{Re}\langle M(x)-w, \eta(y, x)\rangle \\
& +h(y, x)-h(x, x)>0\}
\end{aligned}
$$

is open in $X$.

Then there exists $\hat{y} \in X$ such that

a) $\hat{y} \in S(\hat{y})$ and

b) there exists $\hat{w} \in T(\hat{y})$ with

$$
\operatorname{Re}\langle M(\hat{y})-\hat{w}, \eta(\hat{y}, x)\rangle+h(\hat{y}, x)-h(x, x) \leq 0
$$

for all $x \in S(\hat{y})$.

Moreover if $S(x)=X$ for all $x \in X, E$ is not required to be locally convex and if $T \equiv 0$, the continuity assumption on $\langle\cdot, \cdot\rangle$ can be weakened to the assumption that for each $f \in F$, the map $x \rightarrow\langle f, x\rangle$ is continuous on $X$.

Proof. We divide the proof into three steps.

Step 1. There exists $\hat{y} \in X$ such that $\hat{y} \in S(\hat{y})$ and

$$
\begin{aligned}
& \sup _{x \in S(\hat{y})}\left[\inf _{w \in T(\hat{y})} \operatorname{Re}\langle M(x)-w, \eta(\hat{y}, x)\rangle\right. \\
& +h(\hat{y}, x)-h(x, x)] \leq 0 .
\end{aligned}
$$

Contrary suppose that for each $y \in X$, either $y \notin S(y)$ or there exists $x \in S(y)$ such that

$$
\inf _{w \in T(y)} \operatorname{Re}\langle M(x)-w, \eta(y, x)\rangle+h(y, x)-h(x, x)>0,
$$

that is for each $y \in X$ either $y \notin S(y)$ or $y \in \Sigma$. If $y \notin S(y)$, then by a Hahn-Banach separation theorem for convex sets is locally convex Hausdorff topological vector spaces, there exists $p \in E^{*}$ such that

$$
\operatorname{Re}\langle p, y\rangle-\sup _{x \in S(y)} \operatorname{Re}\langle p, x\rangle>0 \text {. }
$$

For each $p \in E^{*}$, set

$$
V_{p}=\left\{y \in X: \operatorname{Re}\langle p, y\rangle-\sup _{x \in S(y)} \operatorname{Re}\langle p, x\rangle>0\right\} .
$$

Then $V_{p}$ is open in $X$ by Lemma 1 and $\Sigma$ is open in $X$ by hypothesis. Now $X=\Sigma \cup \bigcup_{p \in E^{*}} V_{p}$ and

$\left\{\Sigma, V_{p}: p \in E^{*}\right\}$ is an open covering for $X$. Since $X$ is compact subset of $E$, there exists $p_{1}, p_{2}, \cdots, p_{n} \in E^{*}$ such that $X=\Sigma \cup \bigcup_{i=1}^{n} V_{p_{i}}$ for $i=1,2, \cdots, n$. Let $V_{i}=V_{p_{i}}$ for $i=1,2, \cdots, n$ and $\left\{\beta_{0}, \beta_{1}, \cdots, \beta_{n}\right\}$ be a continuous partition of unity on $X$ subordinated to the 
covering $\left\{V_{0}, V_{1}, \cdots, V_{n}\right\}$. Then $\beta_{0}, \beta_{1}, \cdots, \beta_{n}$ are continuous non-negative real valued functions on $X$ such that $\beta_{i}$ vanishes on $X \backslash V_{i}$ for each $i=0,1, \cdots, n$ and $\sum_{i=0}^{n} \beta_{i}(x)=1$ for all $x \in X$ (see [17] p. 83).

Define $\varphi: X \times X \rightarrow \mathbb{R}$ by

$$
\begin{aligned}
& \varphi(x, y)=\beta_{0}(y) \\
& {\left[\inf _{w \in T(y)} \operatorname{Re}\langle M(x)-w, \eta(y, x)\rangle+h(y, x)-h(x, x)\right]} \\
& +\sum_{i=1}^{n} \beta_{i}(y) \operatorname{Re}\left\langle p_{i}, \eta(y, x)\right\rangle
\end{aligned}
$$

for each $x, y \in X$. Then we have

1) $E$ is Hausdorff for each $A \in F(X)$ and each fixed $x \in \operatorname{co}(A)$ the map

$$
y \rightarrow \inf _{w \in T(y)} \operatorname{Re}\langle M(x)-w, \eta(y, x)\rangle+h(y, x)-h(x, x)
$$

is lower semicontinuous on $\operatorname{co}(A)$ by Lemma 3 and the fact that $h$ is continuous on $\operatorname{co}(A)$, therefore the map

$$
\begin{aligned}
& y \rightarrow \beta_{0}(y) \\
& {\left[\inf _{w \in T(y)} \operatorname{Re}\langle M(x)-w, \eta(y, x)\rangle+h(y, x)-h(x, x)\right]}
\end{aligned}
$$

is lower semicontinuous on $\operatorname{co}(A)$ by Lemma 2. Also for each fixed $x \in X$,

$$
y \rightarrow \sum_{i=1}^{n} \beta_{i}(y) \operatorname{Re}\left\langle p_{i}, \eta(y, x)\right\rangle
$$

is continuous on $X$. Hence for each $A \in F(X)$ and each fixed $x \in c o(A)$, the map $y \rightarrow \varphi(x, y)$ is lower semicontinuous on $\operatorname{co}(A)$.

2) for each $A \in F(X)$ and each $y \in c o(A)$, $\min _{x \in A} \varphi(x, y) \leq 0$. Indeed, if these were false then for some $A=\left\{x_{1}, x_{2}, \cdots, x_{n}\right\} \in F(X)$ and some $y \in \operatorname{co}(A)$ (say $y=\sum_{i=1}^{n} \lambda_{i} x_{i}$, where $\lambda_{1}, \lambda_{2}, \cdots, \lambda_{n} \geq 0$ with $\left.\sum_{i=1}^{n} \lambda_{i}=1\right)$, we have $\min _{1 \leq i \leq n} \varphi\left(x_{i}, y\right)>0$. Then for each $i=1,2, \cdots, n$,

$$
\beta_{0}(y)\left[\inf _{w \in T(y)} \operatorname{Re}\left\langle M\left(x_{i}\right)-w, \eta\left(y, x_{i}\right)\right\rangle+h\left(y, x_{i}\right)-h\left(x_{i}, x_{i}\right)\right]+\sum_{i=1}^{n} \beta_{i}(y)\left\langle p_{i}, \eta\left(y, x_{i}\right)\right\rangle>0
$$

So that

$$
\begin{aligned}
0= & \varphi(y, y)=\beta_{0}(y)\left[\inf _{w \in T(y)} \operatorname{Re}\left\langle M\left(\sum_{i=1}^{n} \lambda_{i} x_{i}\right)-w, \eta\left(y, \sum_{i=1}^{n} \lambda_{i} x_{i}\right)\right\rangle+h\left(y, \sum_{i=1}^{n} \lambda_{i} x_{i}\right)-h\left(\sum_{i=1}^{n} \lambda_{i} x_{i}, \sum_{i=1}^{n} \lambda_{i} x_{i}\right)\right] \\
& +\sum_{i=1}^{n} \beta_{i}(y) \operatorname{Re}\left\langle p_{i}, \eta\left(y, \sum_{i=1}^{n} \lambda_{i} x_{i}\right)\right\rangle \\
= & \beta_{0}(y)\left[\inf _{w \in T(y)} \operatorname{Re}\left\langle\sum_{i=1}^{n} \lambda_{i} M\left(x_{i}\right)-w, \eta\left(y, \sum_{i=1}^{n} \lambda_{i} x_{i}\right)\right\rangle+h\left(y, \sum_{i=1}^{n} \lambda_{i} x_{i}\right)-h\left(\sum_{i=1}^{n} \lambda_{i} x_{i}, \sum_{i=1}^{n} \lambda_{i} x_{i}\right)\right] \\
& +\sum_{i=1}^{n} \beta_{i}(y) \operatorname{Re}\left\langle p_{i}, \eta\left(y, \sum_{i=1}^{n} \lambda_{i} x_{i}\right)\right\rangle \\
\geq & \sum_{i=1}^{n} \lambda_{i}\left(\beta_{0}(y)\left[\inf _{w \in T(y)} \operatorname{Re}\left\langle M\left(x_{i}\right)-w, \eta\left(y, x_{i}\right)\right\rangle+h\left(y, x_{i}\right)-h\left(\sum_{i=1}^{n} \lambda_{i} x_{i}, \sum_{i=1}^{n} \lambda_{i} x_{i}\right)\right]+\sum_{i=1}^{n} \beta_{i}(y) \operatorname{Re}\left\langle p_{i}, \eta\left(y, x_{i}\right)\right\rangle\right)>0
\end{aligned}
$$

which is a contradiction.

Thus we have $\min \varphi(x, y) \leq 0$ for each $A \in F(x)$ and each $y \in \operatorname{co}(A)$.

3) Suppose that $A \in F(X), \quad x, y \in c o(A)$ and $\left\{y_{\alpha}\right\}_{\alpha \in \Gamma}$ is a net in $X$ converging to $y$ with $\varphi\left(t x+(1-t) y, y_{\alpha}\right) \leq 0$ for all $\alpha \in \Gamma, t \in[0,1]$.

Case 1. $\beta_{0}(y)=0$.

Note that $\beta_{0}\left(y_{\alpha}\right) \geq 0$ for each $\alpha \in \Gamma$ and $\beta_{0}\left(y_{\alpha}\right) \rightarrow 0$. Since $T(X)$ is strongly bounded and $\left\{y_{\alpha}\right\}_{\alpha \in \Gamma}$ is a bounded net, therefore

$$
\lim \sup _{\alpha}\left[\beta_{0}\left(y_{\alpha}\right)\left(\min _{w \in T\left(y_{\alpha}\right)} \operatorname{Re}\left\langle M(x)-w, \eta\left(y_{\alpha}, x\right)\right\rangle+h\left(y_{\alpha}, x\right)-h(x, x)\right)\right]=0 .
$$

Also

Thus

$$
\beta_{0}(y)\left[\min _{w \in T(y)} \operatorname{Re}\langle M(x)-w, \eta(y, x)\rangle+h(y, x)-h(x, x)\right]=0 .
$$




$$
\begin{aligned}
& \underset{\alpha}{\limsup }\left[\beta_{0}\left(y_{\alpha}\right)\left(\min _{w \in T\left(y_{\alpha}\right)} \operatorname{Re}\left\langle M(x)-w, \eta\left(y_{\alpha}, x\right)\right\rangle+h\left(y_{\alpha}, x\right)-h(x, x)\right)\right]+\sum_{i=1}^{n} \beta_{i}(y) \operatorname{Re}\left\langle p_{i}, \eta(y, x)\right\rangle \\
& =\sum_{i=1}^{n} \beta_{i}(y) \operatorname{Re}\left\langle p_{i}, \eta(y, x)\right\rangle \text { by (1) } \\
& =\beta_{0}(y)\left[\min _{w \in T(y)} \operatorname{Re}\langle M(x)-w, \eta(y, x)\rangle+h(y, x)-h(x, x)\right]+\sum_{i=1}^{n} \beta_{i}(y) \operatorname{Re}\left\langle p_{i}, \eta(y, x)\right\rangle .
\end{aligned}
$$

When $t=1$, we have $\varphi\left(x, y_{\alpha}\right) \leq 0$ for all $\alpha \in \Gamma$ i.e.,

$$
\beta_{0}\left(y_{\alpha}\right)\left[\min _{w \in T\left(y_{\alpha}\right)} \operatorname{Re}\left\langle M(x)-w, \eta\left(y_{\alpha}, x\right)\right\rangle+h\left(y_{\alpha}, x\right)-h(x, x)\right]+\sum_{i=1}^{n} \beta_{i}\left(y_{\alpha}\right) \operatorname{Re}\left\langle p_{i}, \eta\left(y_{\alpha}, x\right)\right\rangle \leq 0
$$

for all $\alpha \in \Gamma$.

Therefore by (3), we have

$$
\begin{aligned}
& \operatorname{lim\operatorname {sup}_{\alpha }}\left[\beta_{0}\left(y_{\alpha}\right) \min _{w \in T\left(y_{\alpha}\right)} \operatorname{Re}\left\langle M(x)-w, \eta\left(y_{\alpha}, x\right)\right\rangle+h\left(y_{\alpha}, x\right)-h(x, x)\right]+\liminf _{\alpha}\left[\sum_{i=1}^{n} \beta_{i}\left(y_{\alpha}\right) \operatorname{Re}\left\langle p_{i}, \eta\left(y_{\alpha}, x\right)\right\rangle\right] \\
& \leq \operatorname{lim\operatorname {sup}_{\alpha }}\left[\beta_{0}\left(y_{\alpha}\right) \min _{w \in T\left(y_{\alpha}\right)} \operatorname{Re}\left\langle M(x)-w, \eta\left(y_{\alpha}, x\right)\right\rangle+h\left(y_{\alpha}, x\right)-h(x, x)+\sum_{i=1}^{n} \beta_{i}\left(y_{\alpha}\right) \operatorname{Re}\left\langle p_{i}, \eta\left(y_{\alpha}, x\right)\right\rangle\right] \leq 0 .
\end{aligned}
$$

Thus

$$
\lim \sup _{\alpha}\left[\beta_{0}\left(y_{\alpha}\right) \min _{w \in T\left(y_{\alpha}\right)} \operatorname{Re}\left\langle M(x)-w, \eta\left(y_{\alpha}, x\right)\right\rangle+h\left(y_{\alpha}, x\right)-h(x, x)\right]+\sum_{i=1}^{n} \beta_{i}(y) \operatorname{Re}\left\langle p_{i}, \eta(y, x)\right\rangle \leq 0 .
$$

Hence by (2) and (4), we have $\varphi(x, y) \leq 0$.

Case 2. $\beta_{0}(y)>0$.

Since $\beta_{0}\left(y_{\alpha}\right) \rightarrow \beta_{0}(y)$, there exists $\lambda \in \Gamma$ such $\varphi\left(y, y_{\alpha}\right) \leq 0$ for all $\alpha \in \Gamma$, i.e.,

$$
\beta_{0}\left(y_{\alpha}\right)\left[\inf _{w \in T\left(y_{\alpha}\right)} \operatorname{Re}\left\langle M(y)-w, \eta\left(y_{\alpha}, y\right)\right\rangle+h\left(y_{\alpha}, y\right)-h(y, y)\right]+\sum_{i=1}^{n} \beta_{i}\left(y_{\alpha}\right) \operatorname{Re}\left\langle p_{i}, \eta\left(y_{\alpha}, y\right)\right\rangle \leq 0
$$

for all $\alpha \in \Gamma$.

Thus

$$
\lim \sup _{\alpha}\left[\beta_{0}\left(y_{\alpha}\right)\left(\inf _{w \in T\left(y_{\alpha}\right)} \operatorname{Re}\left\langle M(y)-w, \eta\left(y_{\alpha}, y\right)\right\rangle+h\left(y_{\alpha}, y\right)-h(y, y)\right)+\sum_{i=1}^{n} \beta_{i}\left(y_{\alpha}\right) \operatorname{Re}\left\langle p_{i}, \eta\left(y_{\alpha}, y\right)\right\rangle\right] \leq 0 .
$$

Hence

$$
\begin{aligned}
& \operatorname{lim\operatorname {sup}_{\alpha }}\left[\beta_{0}\left(y_{\alpha}\right)\left(\inf _{w \in T\left(y_{\alpha}\right)} \operatorname{Re}\left\langle M(y)-w, \eta\left(y_{\alpha}, y\right)\right\rangle+h\left(y_{\alpha}, y\right)-h(y, y)\right)\right]+\liminf _{\alpha}\left[\sum_{i=1}^{n} \beta_{i}\left(y_{\alpha}\right) \operatorname{Re}\left\langle p_{i}, \eta\left(y_{\alpha}, y\right)\right\rangle\right] \\
& \leq \operatorname{lim\operatorname {sup}_{\alpha }}\left[\beta_{0}\left(y_{\alpha}\right)\left(\inf _{w \in T\left(y_{\alpha}\right)} \operatorname{Re}\left\langle M(y)-w, \eta\left(y_{\alpha}, y\right)\right\rangle+h\left(y_{\alpha}, y\right)-h(y, y)\right)+\sum_{i=1}^{n} \beta_{i}\left(y_{\alpha}\right) \operatorname{Re}\left\langle p_{i}, \eta\left(y_{\alpha}, y\right)\right\rangle\right] \leq 0 \text { (by (5)). }
\end{aligned}
$$

Since

$$
\underset{\alpha}{\liminf }\left[\sum_{i=1}^{n} \beta_{i}\left(y_{\alpha}\right) \operatorname{Re}\left\langle p_{i}, \eta\left(y_{\alpha}, y\right)\right\rangle\right]=0
$$

we have

$$
\limsup _{\alpha}\left[\beta_{0}\left(y_{\alpha}\right)\left(\min _{w \in T\left(y_{\alpha}\right)} \operatorname{Re}\left\langle M(y)-w, \eta\left(y_{\alpha}, y\right)\right\rangle+h\left(y_{\alpha}, y\right)-h(y, y)\right)\right] \leq 0 .
$$


Since $\beta_{0}\left(y_{\alpha}\right)>0$ for all $\alpha>\lambda$. It follows that

$$
\begin{aligned}
& \beta_{0}\left(y_{\alpha}\right) \lim \sup _{\alpha}\left[\min _{w \in T\left(y_{\alpha}\right)} \operatorname{Re}\left\langle M(y)-w, \eta\left(y_{\alpha}, y\right)\right\rangle+h\left(y_{\alpha}, y\right)-h(y, y)\right] \\
& =\lim \sup _{\alpha}\left[\beta_{0}\left(y_{\alpha}\right)\left(\min _{w \in T\left(y_{\alpha}\right)} \operatorname{Re}\left\langle M(y)-w, \eta\left(y_{\alpha}, y\right)\right\rangle+h\left(y_{\alpha}, y\right)-h(y, y)\right)\right] .
\end{aligned}
$$

Since $\beta_{0}(y)>0$ by (6) and (7), we have

$$
\lim \sup _{\alpha}\left[\min _{w \in T\left(y_{\alpha}\right)} \operatorname{Re}\left\langle M(y)-w, \eta\left(y_{\alpha}, y\right)\right\rangle+h\left(y_{\alpha}, y\right)-h(y, y)\right] \leq 0 .
$$

Since $T$ is $(\eta, h)$-quasi pseudomonotone operator, we have

$$
\begin{aligned}
& \underset{\alpha}{\limsup }\left[\min _{w \in T\left(y_{\alpha}\right)} \operatorname{Re}\left\langle M(x)-w, \eta\left(y_{\alpha}, x\right)\right\rangle+h\left(y_{\alpha}, x\right)-h(x, x)\right] \\
& \geq \min _{w \in T\left(y_{\alpha}\right)} \operatorname{Re}\langle M(x)-w, \eta(y, x)\rangle+h(y, x)-h(x, x) \text { for all } x \in X .
\end{aligned}
$$

Since $\beta_{0}(y)>0$, we have

$$
\begin{aligned}
& \beta_{0}(y)\left[\limsup _{\alpha}\left(\min _{w \in T\left(y_{\alpha}\right)} \operatorname{Re}\left\langle M(x)-w, \eta\left(y_{\alpha}, x\right)\right\rangle+h\left(y_{\alpha}, x\right)-h(x, x)\right)\right] \\
& \geq \beta_{0}(y)\left[\min _{w \in T(y)} \operatorname{Re}\langle M(x)-w, \eta(y, x)\rangle+h(y, x)-h(x, x)\right] .
\end{aligned}
$$

Thus

$$
\begin{aligned}
& \beta_{0}(y)\left[\limsup _{\alpha}\left(\min _{w \in T\left(y_{\alpha}\right)} \operatorname{Re}\left\langle M(x)-w, \eta\left(y_{\alpha}, x\right)\right\rangle+h\left(y_{\alpha}, x\right)-h(x, x)\right)\right]+\sum_{i=1}^{n} \beta_{i}(y) \operatorname{Re}\left\langle p_{i}, \eta(y, x)\right\rangle \\
& \geq \beta_{0}(y)\left[\min _{w \in T(y)} \operatorname{Re}\langle M(x)-w, \eta(y, x)\rangle+h(y, x)-h(x, x)\right]+\sum_{i=1}^{n} \beta_{i}(y) \operatorname{Re}\left\langle p_{i}, \eta(y, x)\right\rangle .
\end{aligned}
$$

When $t=1$, we have $\varphi\left(x, y_{\alpha}\right) \leq 0$ for all $\alpha \in \Gamma$, i.e.,

$$
\beta_{0}\left(y_{\alpha}\right)\left[\min _{w \in T\left(y_{\alpha}\right)} \operatorname{Re}\left\langle M(x)-w, \eta\left(y_{\alpha}, x\right)\right\rangle+h\left(y_{\alpha}, x\right)-h(x, x)\right]+\sum_{i=1}^{n} \beta_{i}\left(y_{\alpha}\right) \operatorname{Re}\left\langle p_{i}, \eta\left(y_{\alpha}, x\right)\right\rangle \leq 0
$$

for all $\alpha \in \Gamma$.

Thus

$$
\begin{aligned}
0 & \geq \lim \sup _{\alpha}\left[\beta_{0}\left(y_{\alpha}\right) \min _{w \in T\left(y_{\alpha}\right)} \operatorname{Re}\left\langle M(x)-w, \eta\left(y_{\alpha}, x\right)\right\rangle+h\left(y_{\alpha}, x\right)-h(x, x)+\sum_{i=1}^{n} \beta_{i}\left(y_{\alpha}\right) \operatorname{Re}\left\langle p_{i}, \eta\left(y_{\alpha}, x\right)\right\rangle\right] \\
& \geq \lim \sup _{\alpha}\left[\beta_{0}\left(y_{\alpha}\right) \min _{w \in T\left(y_{\alpha}\right)} \operatorname{Re}\left\langle M(x)-w, \eta\left(y_{\alpha}, x\right)\right\rangle+h\left(y_{\alpha}, x\right)-h(x, x)\right]+\liminf _{\alpha}\left[\sum_{i=1}^{n} \beta_{i}\left(y_{\alpha}\right) \operatorname{Re}\left\langle p_{i}, \eta\left(y_{\alpha}, x\right)\right\rangle\right] \\
& =\beta_{0}(y)\left[\limsup _{\alpha}\left\{\min _{w \in T\left(y_{\alpha}\right)} \operatorname{Re}\left\langle M(x)-w, \eta\left(y_{\alpha}, x\right)\right\rangle+h\left(y_{\alpha}, x\right)-h(x, x)\right\}\right]+\sum_{i=1}^{n} \beta_{i}(y) \operatorname{Re}\left\langle p_{i}, \eta(y, x)\right\rangle \\
& \geq \beta_{0}(y)\left[\min _{w \in T(y)} \operatorname{Re}\langle M(x)-w, \eta(y, x)\rangle+h(y, x)-h(x, x)\right]+\sum_{i=1}^{n} \beta_{i}(y) \operatorname{Re}\left\langle p_{i}, \eta(y, x)\right\rangle \text { (by (8)). }
\end{aligned}
$$

Hence, we have $\varphi(x, y) \leq 0$.

Since $X$ is a compact subset of the Hausdorff topological vector space $E$, it is also closed. Now if we take $K=X$, then for any $x_{0} \in K=X$, we have $\varphi\left(x_{0}, y\right)>0$ for all $y \in X \backslash K(=X \backslash X=\varnothing)$.

Thus $\varphi$ satisfies all the hypothesis of Theorem 1 . Hence by Theorem 1 , there exists $\hat{y} \in K$ such that 


$$
\begin{gathered}
\varphi(x, \hat{y}) \leq 0 \text { for all } x \in X, \\
\beta_{0}(\hat{y})\left[\inf _{w \in T(\hat{y})} \operatorname{Re}\langle M(x)-w, \eta(\hat{y}, x)\rangle+h(\hat{y}, x)-h(x, x)\right] \\
+\sum_{i=1}^{n} \beta_{i}(\hat{y}) \operatorname{Re}\left\langle p_{i}, \eta(\hat{y}, x)\right\rangle \leq 0 \text { for all } x \in X .
\end{gathered}
$$

Now the rest of the proof of Step 1 is similar to the proof in Step 1 of Theorem 1 in [11]. Hence Step 1 is proved.

Step 2.

$$
\begin{aligned}
& \inf _{w \in T(\hat{y})} \operatorname{Re}\langle M(\hat{y})-w, \eta(\hat{y}, x)\rangle+h(\hat{y}, x)-h(x, x) \leq 0 \\
& \text { for all } x \in S(y) .
\end{aligned}
$$

From Step 1, we have $\hat{y} \in S(\hat{y})$ and

$$
\begin{aligned}
& \inf _{w \in T(\hat{y})} \operatorname{Re}\langle M(x)-w, \eta(\hat{y}, x)\rangle+h(\hat{y}, x)-h(x, x) \leq 0 \\
& \text { for all } x \in S(y) .
\end{aligned}
$$

Since $S(\hat{y})$ is a convex subset of $X$ and $M$ is linear, continuous along line segments in $X$, by Lemma 4 we have

$$
\begin{aligned}
& \left.\inf _{w \in T(\hat{y})} \operatorname{Re}\langle M(\hat{y})-w, \eta(\hat{y}, x)\rangle+h(\hat{y}, x)-h(x, x)\right) \leq 0 \\
& \text { for all } x \in S(y) .
\end{aligned}
$$

Step 3. There exists $\hat{w} \in T(\hat{y})$ with

$$
\begin{aligned}
& \operatorname{Re}\langle M(\hat{y})-\hat{w}, \eta(\hat{y}, x)\rangle+h(\hat{y}, x)-h(x, x) \leq 0 \\
& \text { for all } x \in S(y) .
\end{aligned}
$$

By Step 2 and applying Theorem 2 as proved in Step 3 of Theorem 1 in [11], we can show that there exists $\hat{w} \in T(\hat{y})$ such that

$$
\operatorname{Re}\langle M(\hat{y})-\hat{w}, \eta(\hat{y}, x)\rangle+h(\hat{y}, x)-h(x, x) \leq 0
$$

for all $x \in S(y)$.

We observe from the above proof that the requirement that $E$ be locally convex is needed when and only when the separation theorem is applied to the case $y \notin S(y)$. Thus if $S: X \rightarrow 2^{X}$ is the constant map $S(x)=X$ for all $x \in X, E$ is not required to be locally convex.

Finally, if $T \equiv 0$, in order to show that for each $x \in X, y \rightarrow \varphi(x, y)$ is lower semicontinuous, Lemma 3 is no longer needed and the weaker continuity assumption as $\langle\cdot, \cdot\rangle$ that for each $f \in E$, the map $x \rightarrow\langle f, x\rangle$ is continuous on $X$ is sufficient. This completes the proof.

\section{REFERENCES}

[1] F. Giannessi, "Theorems of Alternative Quadratic Pro- grams and Complementarity Problems,” In: R. W. Cottle, F. Gianessi and J. L. Lions, Eds., Variational Inequalities and Complementarity Problems, John Wiley and Sons, Chichester, 1980.

[2] D. Kinderlehrer and G. Stampacchia, “An Introduction to Variational Inequalities and Their Applications in Pure and Applied Mathematics," Vol. 88, Academic Press, New York, 1980.

[3] F. E. Browdev, "Existence and Approximation of Solutions of Nonlinear Variational Inequalities,” Proceedings of the National Academy of Sciences of the United States of America, Vol. 56, No. 4, 1966, pp. 1080-1086.

[4] G. Y. Chen and G. M. Cheng, "Vector Variational Inequality and Vector Optimizations," Lecture Notes in Economics and Mathematical Systems, Vol. 285, 1967, pp. 408-456.

[5] M. S. R. Chowdhury and K. K. Tan, "Generalization of Ky Fan’s Minimax Inequality with Applications to Generalized Variational Inequalities for Pseudomonotone Operators and Fixed Point Theorems," Journal of Mathematical Analysis and Applications, Vol. 204, No. 3, 1996, pp. 910-929. doi:10.1006/jmaa.1996.0476

[6] M. S. R. Chowdhury, "The Surjectivity of Upper Hemicontinuous and Pseudomonotone Type II Operators in Reflexive Banach Ppaces," Journal Bangladesh Mathematical Society, Vol. 20, 2000, pp. 45-53.

[7] H. Brezis, L. Nirenberg and G. Stampacchia, "A Remark on Ky Fan's Minimax Principle,” Bollettino Unione Matematica Italiana, Vol. 6, No. 4, 1972, pp. 293-300.

[8] K. Fan, “A Minimax Inequality and Applications,” In: O. Shisha, Ed., Inequalities III, Academic Press, San Diego, 1972, pp. 103-113.

[9] M. S. R. Chowdhury and K. K. Tan, "Generalized Variational Inequalities for Quasimonotone Operators and Applications,” Bulletin of Polish Academy of Science, Vol. 45, No. 1, 1997, pp. 25-54.

[10] M. H. Shih and K. K. Tan, "Generalized Bi-Quasi Variational Inequalities," Journal of Mathematical Analysis and Applications, Vol. 143, No. 1, 1989, pp. 66-85. doi:10.1016/0022-247X(89)90029-2

[11] M. S. R. Chowdhury and K. K. Tan, “Applications of Upper Hemicontinuous Operators on Generalized Bi-Quasi Variational Inequalities in Locally Convex Topological Vector Spaces,” Positivity, Vol. 3, No. 4, 1999, pp. 333344. doi:10.1023/A:1009849400516

[12] M. S. R. Chowdhury and K. K. Tan, “Applications of Pseudomonotone Operators with Some Kind of Upper Semicontinuity in Generalized Quasi Variational Inequalities on Noncompact Sets," Proceeding of American Mathematica Society, Vol. 126, No. 10, 1998, pp. 2957 2968. doi:10.1090/S0002-9939-98-04436-0

[13] X. P. Ding and E. Tarafdar, “Generalized Variational Like Inequalities with Pseudomonotone Setvalued Mappings," Archieve Journal of Mathematics, Vol. 74, No. 4, 2000, pp. 302-313. doi:10.1007/s000130050447

[14] M. H. Shih and K. K. Tan, "Generalized Quasi Variational Inequalities in Locally Convex Topological Vector Spaces," Journal of Mathematical Analysis and Applica- 
tions, Vol. 108, No. 2, 1985, pp. 333-343. doi:10.1016/0022-247X(85)90029-0

[15] W. Takahashi, "Nonlinear Variational Inequalities and Fixed Point Theorem," Journal of Mathematical Society of Japan, Vol. 28, No. 1, 1976, pp. 168-181. doi:10.2969/jmsj/02810168

[16] H. Kneser, "Sur un Theoreme Fundamental de la Theorie des Jeux,” CRAS Paris, Vol. 234, 1952, pp. 2418-2420.

[17] R. T. Rockafeller, “Convex Analysis,” Princeton University Press, Princeton, 1970. 